\title{
Urbanizaciones y espacios públicos en Lima: San Isidro, Chorrillos y Miramar. 1919-1930*
}

\author{
Urbanizations and public spaces in Lima: \\ San Isidro, Chorrillos and Miramar. 1919-1930
}

Fredy Quispe**

Investigador independiente Recibido: 8 de octubre de 2020 Aceptado: 16 de noviembre de 2020

\section{RESUMEN}

La ciudad de Lima tuvo un acelerado desarrollo urbano durante el Oncenio de Leguía (1919-1930). El área urbana se duplicó y el triángulo conformado entre Lima, Callao y Chorrillos se empezó a ocupar. Las avenidas Leguía, El Progreso y Costanera consolidaron el desarrollo urbano y se proyectaron más de treinta urbanizaciones. San Isidro, Chorrillos y Miramar se construyeron en este periodo y estuvieron orientadas a distintos niveles socioeconómicos. La urbanización San Isidro se encuentra rodeando el bosque del Olivar, la urbanización Chorrillos tiene como referente al morro solar y la urbanización Miramar se presenta frente al océano Pacífico Sur.

Las urbanizaciones desarrolladas durante este periodo no proyectaban nuevas áreas destinadas a espacios públicos ni áreas verdes y pueden explicar, en parte, la actual ausencia de plazas, parques, zonas recreación y espacios de afirmación ciudadana que adolece la capital.

Palabras clave: urbanizaciones, espacios públicos, Lima, Oncenio de Leguía, San Isidro, Chorrillos, Miramar.

\begin{abstract}
The city of Lima had an accelerated urban development during the Oncenio of Leguía (19191930). The urban area doubled and the triangle formed between Lima, Callao and Chorrillos began to be occupied. Leguía, Costanera and El Progreso avenues consolidated urban development and more than thirty urbanizations were projected. San Isidro, Chorrillos and Miramar were built in this period and were oriented to different socioeconomic levels. The San Isidro urbanization is located around the Olivar forest, the Chorrillos urbanization has as a reference the solar hill and the Miramar urbanization faces the ocean South Pacific.

The urbanizations developed during this period did not project new areas for public spaces or green areas, and may partly explain the current absence of squares, parks, recreation areas and spaces for citizen affirmation that the capital suffers from.
\end{abstract}

Keywords: urbanizations, public spaces, Lima, Oncenio of Leguía, San Isidro, Chorrillos, Miramar.

* Antecedentes del documento. El presente artículo forma parte de un capítulo de la Tesis de Maestría desarrollada por el autor, cuyo título es: La revista Ciudad y Campo. 1924-1930.

** Fredy Quispe Aguilar. Arquitecto por la Pontificia Universidad Católica del Perú. Egresado de la Maestría en Ciencias con mención en Arquitectura - Historia, Teoría y Crítica de la Universidad Nacional de Ingeniería. 


\section{Introducción}

El Oncenio de Leguía (1919-1930) fue un periodo sensible en el desarrollo urbano de la ciudad de Lima. En esta etapa se dio la primera expansión de la capital, llegando a duplicarse el área urbana. El triángulo conformado entre Lima, Callao y Chorrillos se consolidó a partir de la construcción de las avenidas Leguía, Costanera y El Progreso. La avenida Leguía, inaugurada para las celebraciones del centenario de la independencia, fue la vía más importante y conectó el centro de Lima con los entonces balnearios de Miraflores y Barranco. En esta nueva área urbana se desarrollaron más de treinta nuevas urbanizaciones. Las urbanizaciones, en su mayoría, ocupaban el espacio de antiguas haciendas pertenecientes a la aristocracia limeña.

El siguiente artículo aborda a las urbanizaciones construidas durante este periodo y selecciona las de San Isidro, Chorrillos y Miramar como referentes de tres distintos niveles socioeconómicos, tres distintas ubicaciones y tres distintas formas de trazado y organización. Las urbanizaciones buscaron rentabilizar al máximo sus propiedades y casi no se dejó áreas destinadas a espacios públicos o importantes áreas verdes. Se construyeron vías y calles de entre veinte a cincuenta metros de sección. Las manzanas y los lotes variaban en forma y tamaño. Actualmente, Lima adolece de espacios públicos y, en cierto sentido, puede revelarse su ausencia en el desarrollo de estas urbanizaciones.

\section{Evolución y crecimiento de Lima durante la República (1821-1919)}

Desde el inicio de la República (1821) hasta el comienzo del gobierno de Leguía (1919), Lima atravesó un periodo de evolución y crecimiento de 98 años. Durante este tiempo, el desarrollo de la capital se dio de manera lenta y el inicio del segundo gobierno leguiísta representó la primera gran expansión de la ciudad en el siglo XX. (Figura 1)

Uno de los planos más importantes del siglo XIX fue el realizado en 1859 por el ingeniero y arquitecto Antonio María Dupard. Este plano se publicó en el Atlas Geográfico del Perú y según Juan Gunther (1983): “este plano, por su precisión y por la calidad del dibujo, es el mejor de todos los elaborados en el siglo pasado" (p. 30). Durante los gobiernos de Ramón Castilla (1845-1851 y 1855-1862) y José Rufino Echenique (1851-1855), el Perú y Lima pudieron desarrollarse urbanísticamente gracias a la prosperidad de las industrias del guano y del salitre. "Así, se pavimentaron las calles con adoquines, se implantó la iluminación a gas, se reemplazaron las tuberías para agua y desagüe, y se construyeron los ferrocarriles Lima-Callao y, posteriormente, Lima-Chorrillos" (Hamann, 2015, p. 29).

Entre 1868 y 1872, durante el gobierno de José Balta, la capital siguió expandiéndose y se tomaron medidas para ordenar el crecimiento de la ciudad. Se encargó el primer Plan Regulador de Lima al ingeniero del Estado Luis Sadá y la demolición de las murallas al norteamericano Enrique Meiggs. En 1865 comienza la guerra con España y, en 1879, Perú se enfrenta a Chile. La Guerra del Pacífico concluyó oficialmente el 20 de octubre de 1883 con el Tratado de Ancón y el Perú quedó sumergido en una crisis que paralizó todos los proyectos.

Culminada la guerra, empezó un proceso de reconstrucción nacional. Lima, que se había caracterizado por la estrechez de sus calles, adquirió otras dimensiones con aperturas de calles y avenidas mucho más anchas, tipo bulevares (Harth-terré, 1935). En los años posteriores se trazaron las avenidas Grau y Alfonso Ugarte que reemplazaron las murallas. En 1898, "Lima llegó a una superficie de 1292 hectáreas; con lo que la ciudad creció 185 hectáreas más que en 1857 , según la estadística hecha por Manuel Atanasio Fuentes" (Hamann, 2015, p. 65).

En 1903 la población ascendió a 130289 y en 1908 la cifra llegó a 140000 habitantes. En 1904, Santiago Basurco realizó un plano en el que se señala la avenida La Colmena, la avenida Central y la avenida Grau. En 1913, se construyeron las líneas del ferrocarril a Magdalena y, hacia el sur, las líneas que conectaban Lima con Miraflores y Barranco. Entre 1909 y 1912, Augusto B. Leguía llega por primera vez a la presidencia; en el año 1919 es elegido por segunda vez, dando inicio al denominado Oncenio de Leguía.

En 1919 se efectuó un nuevo censo, la capital contaba con 176000 habitantes y la superficie urbana ocupada llegaba a 1430 hec- 


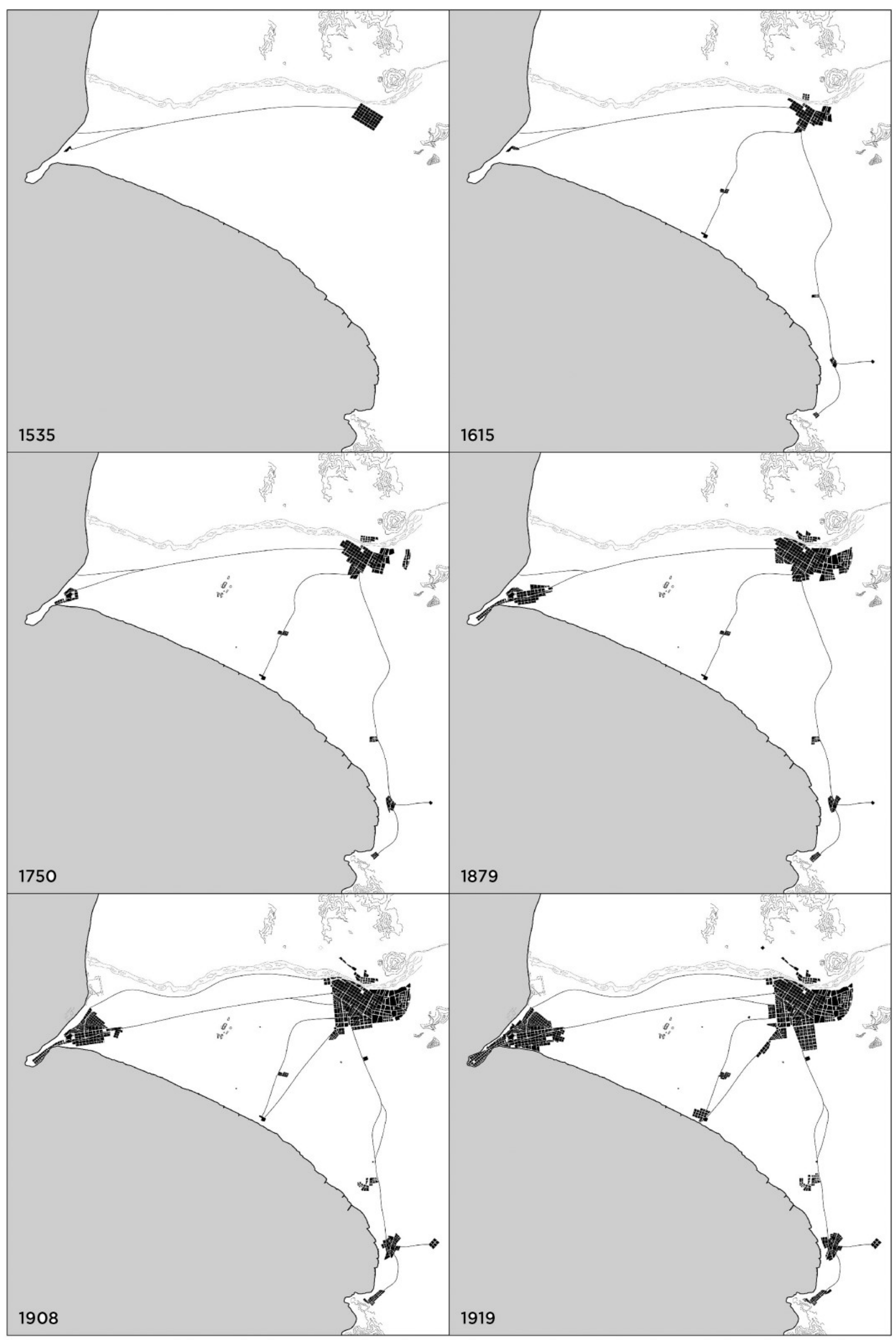

Figura 1. Evolución y desarrollo de Lima (1535-1919)

Elaboración propia (2020). Fuente: Planos de Lima 1613-1983 (1983) 
táreas. Como señala Werner Lange (1927), el crecimiento de la ciudad desde 1535 se dio de manera centripetal y no centrifugal, es decir, el crecimiento se dio en el centro de Lima, el Callao y Chorrillos. Estos tres puntos se conectaron mediante las vías de ferrocarril y se consolidaron con la construcción de las avenidas Leguía y El Progreso.

\section{Lima y el Oncenio de Leguía (1919-1930)}

Augusto B. Leguía inició su segundo gobierno en 1919. La denominada "Patria Nueva" significó el fin de la República Aristocrática (1895-1919) e implicó una voluntad de ruptura con la mentalidad colonial, mantenida por la clase política del siglo XIX. Durante esta etapa, Leguía se propuso mostrar al mundo que el Perú se estaba convirtiendo en un país moderno y tuvo a Lima como su principal espacio de intervención. Como señala Ludeña (2002):

De una u otra forma, casi todos los gobernantes del Perú han tenido en la ciudad de Lima a uno de sus objetos preferidos de intervención. Pero si realmente existe alguien para quien esta relación tuvo el sentido de una relación vital desde el punto de vista no solo de la existencia política, económica o cultural, este es Leguía (p. 40).

En Lima se ejecutaron las principales obras públicas y se impulsó la modernización de la ciudad. Leguía aspiraba a que Lima se convirtiera en "la capital de la costa sur del Pacífico". Esta etapa coincidió, a su vez, con las celebraciones de la independencia del Perú (1921) y la batalla de Ayacucho (1924). Como parte de las festividades se construyeron importantes espacios públicos, monumentos y edificios. Entre ellos se encuentran las plazas San Martín, Sucre y Washington; los monumentos a San Martín, a Washington, a Petit Thouars, a Sucre, al obrero y a Manco Cápac; edificios públicos y privados como el Panteón de los Próceres, el Ministerio de Fomento, el Museo de la Breña, el Country Club, el edificio Rímac, el Hospital Loayza, el edificio Minería, el Palacio Legislativo, el Palacio de Justicia, el Orfelinato Pérez Araníbar, la Escuela de Agricultura y Veterinaria, el Club Nacional, el Hotel Bolívar, entre otros.
El gobierno de Leguía diseñó una política atractiva para la inversión extranjera, especialmente norteamericana. El Perú pasó de tener una larga relación durante años con Inglaterra, a iniciar, de acuerdo con Lawrence A. Clayton, el período de mayor cooperación entre Estados Unidos y Perú (2002). Esto tuvo sus efectos en la llegada de importantes empresas y profesionales extranjeros. Como señalan Bromley y Barbageleta (1945): “El impulso realizador se consigue mediante intervenciones de empresas extranjeras sujetas a obligaciones contractuales y al apoyo prestado en el ramo de las industrias" (p. 104). Esto produjo un cambio en las edificaciones, avenidas, espacios públicos y nuevas urbanizaciones, impulsado por la introducción del concreto armado. Entre las más importantes empresas constructoras que arribaron al Perú se encuentran The Foundation Company y Fred T. Ley \& Co. Ltda. encargadas de las obras más importantes de saneamiento, pavimentación, infraestructura y edificaciones públicas.

Esta etapa también coincidió con un aumento en la población y una reducción de la tasa de mortalidad. Hacia 1919, Lima había registrado una población de 176000 habitantes y en el año 1920 Lima y el Callao contaban con más de 300 000. Hacia el término del oncenio de Leguía en 1930, la población de Lima aumentó a 272742 habitantes y la población de Lima y el Callao ascendió a 442300 . Es decir, hubo un aumento en la población de la ciudad de un $57 \%$ y los habitantes de Lima y el Callao aumentaron en un $47 \%$. El área urbana de Lima pasó de 1020 hectáreas en 1920 a duplicarse a 2037 en 1931. Como menciona Basadre: "Gran parte de la actual estructura urbana de Lima surgió entre 1919 y 1930 o ha provenido del impulso entonces iniciado" (2005, p. 127).

Las nuevas avenidas El Progreso y Leguía consolidaron el crecimiento de la capital. La avenida Leguía, inaugurada en 1921, fue la más importante y determinó la ubicación de las principales urbanizaciones. En 1922 se inició la urbanización del Fundo Santa Beatriz y fue el punto de partida para la aparición de nuevas áreas urbanas. Las nuevas urbanizaciones habían sido fundos de propiedad de las familias aristocráticas de la ciudad, que 
surgieron como respuesta al acelerado incremento de población en Lima para generar cuantiosas ganancias a sus propietarios, ofreciendo la venta de terrenos y chalets. La revista Ciudad y Campo fue uno de los principales medios de difusión y propaganda de estas propiedades. Los terrenos se ofrecían con áreas desde $350 \mathrm{~m} 2$ y podían llegar hasta los $1500 \mathrm{~m} 2$. Los chalets se ofrecían en áreas aproximadas de $300 \mathrm{m2}$, dos pisos, jardín, hall, sala, comedor, 5 dormitorios, baño, cocina, cuarto de sirvientes y corral.

Más de 34 urbanizaciones se proyectaron durante el oncenio de Leguía, entre ellas se encuentran: Plaza 2 de mayo, Santa Beatriz, Av. del Brasil, Av. la Magdalena, Balconcillo, Breña, Cocharcas, Chacra Alta, Chacra Colorada, Villa Olaya o la Chira Chorrillos, El Progreso, El Prado, Garden City, Jesús María, La Condesa, La Herradura, Leuro, Lince y Lobatón, Lima Limitada, Limatambo, Chacarilla, Magdalena, Manzanilla, Miraflores, Miramar, Moyobamba, Orbea, Santa Cruz, San Isidro, Surquillo, San Miguel, Tejada, Tejadita, La Perla y Cía. Parque de la Av. del Brasil.

\section{Urbanizaciones y espacio público}

Entre 1919 y 1930, se proyectaron y construyeron más de treinta urbanizaciones dentro del triángulo conformado entre Lima, Miraflores y Callao. Las principales avenidas consolidaron el desarrollo de la ciudad. Las urbanizaciones presentaban, en su mayoría, el trazado ortogonal y secciones de vías entre veinte y cincuenta metros. La urbanización San Isidro, proyectada por Manuel Piqueras Cotolí, representó un cambio en el urbanismo con su diseño pintoresquista. La urbanización Chorrillos fue de propiedad del hijo de Augusto B. Leguía y la urbanización Miramar se situaba frente al Pacífico Sur. (Figura 2)

\subsection{Urbanización San Isidro}

El espacio ocupado por El Olivar, durante la etapa prehispánica, formó parte de Limac-Ritmaktampu. La planta del olivo llega por primera vez al Perú a cargo de Antonio de Rivera y Gonzalo Pizarro aproximadamente en 1539, desde Sevilla. Durante el trayecto de España al Perú, solo tres ejemplares de olivar llegaron a sobrevivir y adaptarse a las condiciones climáticas del país. Hacia el año 1620 , se planta- ron olivos en lo que entonces se denominaba Hacienda Limatambo. Se data que los frutos fueron cosechados y comercializados como aceite de oliva. Se conservaban en botijas de barro y también fueron utilizados como bálsamo para los santos óleos. Hacia 1730 se tenían registrados 1500 olivos aproximadamente y hacia el año 1828 el número ascendió a 2338. En 1777, la hacienda fue adquirida por Don Isidoro de Abarca y a partir de ahí fue denominada como Los Condes de San Isidro. En 1853, la hacienda pasó a propiedad de José Gregorio Paz Soldán y Ureta. Finalmente, en 1887, su nieta Luisa Paz Soldán se hizo acreedora de la llamada Hacienda de San Isidro y durante esta etapa se plantaron la mayor parte de los olivos centenarios conocidos hoy en día.

En 1920, dentro del periodo gubernamental de Leguía, se formó la Compañía Urbanizadora San Isidro Limitada y se encargó el diseño de la urbanización al arquitecto y escultor español Piqueras Cotolí. La urbanización San Isidro ofrecía terrenos y chalets fuera, al borde y dentro del Bosque del Olivar. En las publicaciones de empresas urbanizadoras de Lima y alrededores, presentadas en la revista Ciudad y Campo, se menciona en una primera instancia y en el año 1925 a José Ortiz de Zevallos como el gerente y propietario de la compañía urbanizadora. Posteriormente, en 1928, se menciona a Manuel Moreyra Paz Soldán como el único gerente general y propietario.

Manuel Celestino Piqueras Cotolí (18851937) fue hijo de padres valencianos. En 1919 llega al Perú como profesor de escultura en la Escuela Nacional de Bellas Artes de Lima, debido a su estrecha relación con el pintor Enrique Domingo Barreda. Se estableció en la capital y se le encargaron importantes obras de escultura, arquitectura y urbanismo. Entre sus obras mas importantes de arquitectura destacan la nueva fachada de la Escuela $\mathrm{Na}$ cional de Bellas Artes (1920-1924) el Pabellón del Perú en la Exposición Iberoamericana de Sevilla (1929), el "Salón Inca" en el Palacio de Gobierno (1924), la tumba a Francisco Pizarro dentro de la catedral de Lima, el monumento a Ricardo Palma dentro del Parque de la Exposición, la estatua de Hipólito Unanue en el Parque Universitario, el diseño de la Plaza San Martín (1921) y el diseño de la urbanización San Isidro. 


\section{ARQUITEXTOS}

ISSN 1819-2939 / ISSNe 2706-8099

Año 27, № 35, enero-diciembre de 2020, 83-96

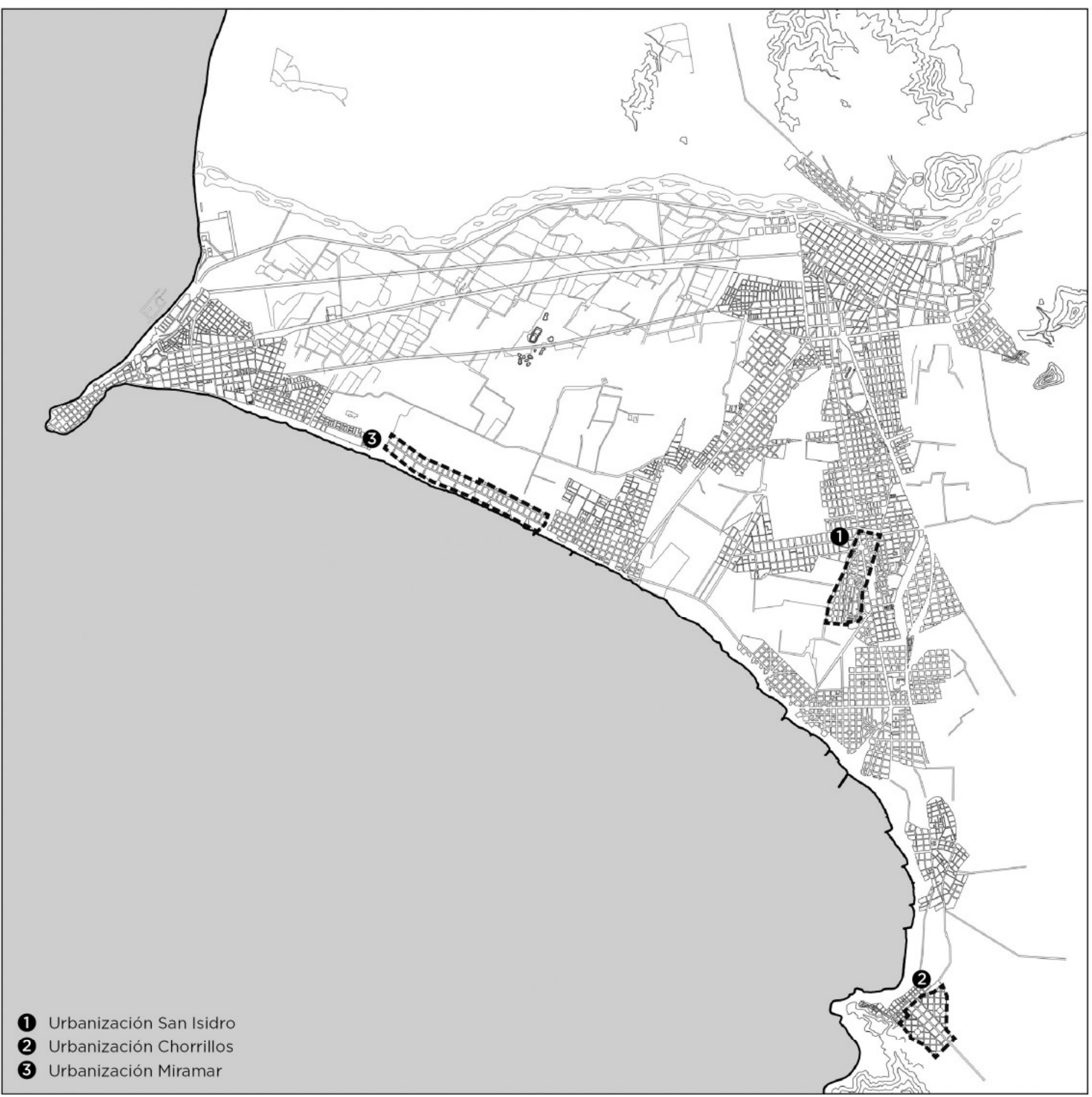

Figura 2. Plano de Lima y urbanizaciones en 1926.

Elaboración propia (2020). Fuente: Ciudad y Campo, $n^{\circ} 23$ (noviembre, 1926) 
La urbanización se caracterizó por incluir al Bosque del Olivar y se encontraba conectada a la ciudad mediante la avenida Leguía. El espacio ocupado por la urbanización San Isidro se encuentra entre el centro de Lima y Miraflores. La avenida Leguía fue inaugurada en 1921 y consolidó el desarrollo de las urbanizaciones ubicadas a lo largo de su tramo. Las mas importantes y costosas propiedades se ubicaron a lo largo de esta crucial avenida. (Figura 3)

\section{Trazado, manzanas y zonificación}

El primer bosquejo de la urbanización San Isidro fue realizado entre 1920 y 1922, contó con 77 manzanas de distintos tamaños y formas que a su vez se interconectaban con el bosque El Olivar. La característica más resaltante de esta urbanización fue el diseño y trazo "pintoresco" realizado por Piqueras Cotolí, con referencias al urbanismo de Camilo Sitte. A diferencia de otras, el trazado no fue ortogonal ni ajedrezado, sino presentaba calles curvas, largas, cortas y diagonales. Como señala Wiley Ludeña: "con la urbanización San Isidro se inicia en el Perú una nueva tradición urbanística en el ámbito de lo residencial. Piqueras abre la posibilidad de recrear un nuevo lenguaje y una espacialidad inusitada para Lima" (2009, p.173).

Las manzanas casi no se repiten, cada una presenta distinta forma y tamaño. Además, distintas relaciones con la ciudad, ya que algunas se encuentran dentro del bosque el Olivar, al borde del bosque, al borde de la avenida Leguía, rodeada de otras manzanas, etc. Cada una de estas diferencias, generó diversidad de relaciones entre la urbanización, la ciudad y el bosque. El nuevo urbanismo formulado por Piqueras Cotolí, como señala Ludeña, apuesta por una dramatización romántica del paisaje y una recusación orgánica de la ortogonalidad y monumentalización centrípeta neobarroca (Ludeña, 2009).

La zonificación fue únicamente residencial; no se proyectó ni pensó en zonas destinadas al comercio, educación, salud, industria ni cultura. El espacio que concentraba los principales edificios comerciales seguía siendo el centro de Lima y los residentes fueron abandonando ese espacio para trasladarse a los nuevos suburbios de la ciudad. La cone- xión con las urbanizaciones residenciales se pensó a partir del automóvil y se consolidó a partir de la construcción y pavimentación de las nuevas avenidas.

\section{Vías, lotes y chalets}

Durante el gobierno de Leguía se impulsaron obras de saneamiento y, como se señala en la revista Ciudad y Campo, la urbanización San Isidro contaba con obras de agua potable, desagüe, vereda y luz eléctrica. Hacia 1924, la mayoría de sus vías se encontraban pavimentadas y los trabajos fueron realizados por la importante constructora The Foundation Company. Las secciones de las vías presentaban anchos variables entre 15 y 40 metros. Solo estuvieron pensadas para el automóvil y no se plantearon paseos peatonales, alamedas o la arborización de las calles.

La irregularidad de las manzanas generó lotes diversos. En su mayoría, la urbanización presentaba lotes de gran tamaño, entre 300 y $1400 \mathrm{~m} 2$, destinados a la clase alta. La venta de los lotes y chalets se publicó en la revista Ciudad y Campo. Se ofrecían propiedades dentro y fuera del bosque de San Isidro. El costo de los terrenos dentro del bosque del Olivar eran los más elevados y se ofrecían áreas entre $800 \mathrm{~m} 2$ y $1000 \mathrm{m2}$. El costo aproximado era de 10 Lp por cada metro cuadrado de terreno. Los lotes fuera del bosque del Olivar contaban con medidas más pequeñas y variaban entre $302 \mathrm{m2}, 581 \mathrm{m2}$ y $1235 \mathrm{m2}$. Los costos eran, aproximadamente, de $6 \mathrm{Lp}$ por $\mathrm{m} 2$ y los chalets se ofrecían de $16 \times 23 \mathrm{~m}$. La distribución consistía en tres dormitorios, baño, comedor, salón, vestíbulo, cuarto de sirvientes, despensa, cocina y jardín. El costo aproximado por cada chalet era de 1650 Lp.

\section{Espacios públicos}

La urbanización San Isidro solo proyectó un nuevo espacio destinado al área verde y espacio público, ya que el bosque del Olivar y la avenida Leguía se encontraban previos al trazo de Piqueras Cotolí. La nueva área verde se ubicó en el óvalo de la urbanización, que intersectaba con la recién inaugurada avenida Leguía. El resto de calles se dedicó al tránsito vehicular y no se destinaron más áreas para plazas y alamedas, ni espacios para la recreación y el ocio. 


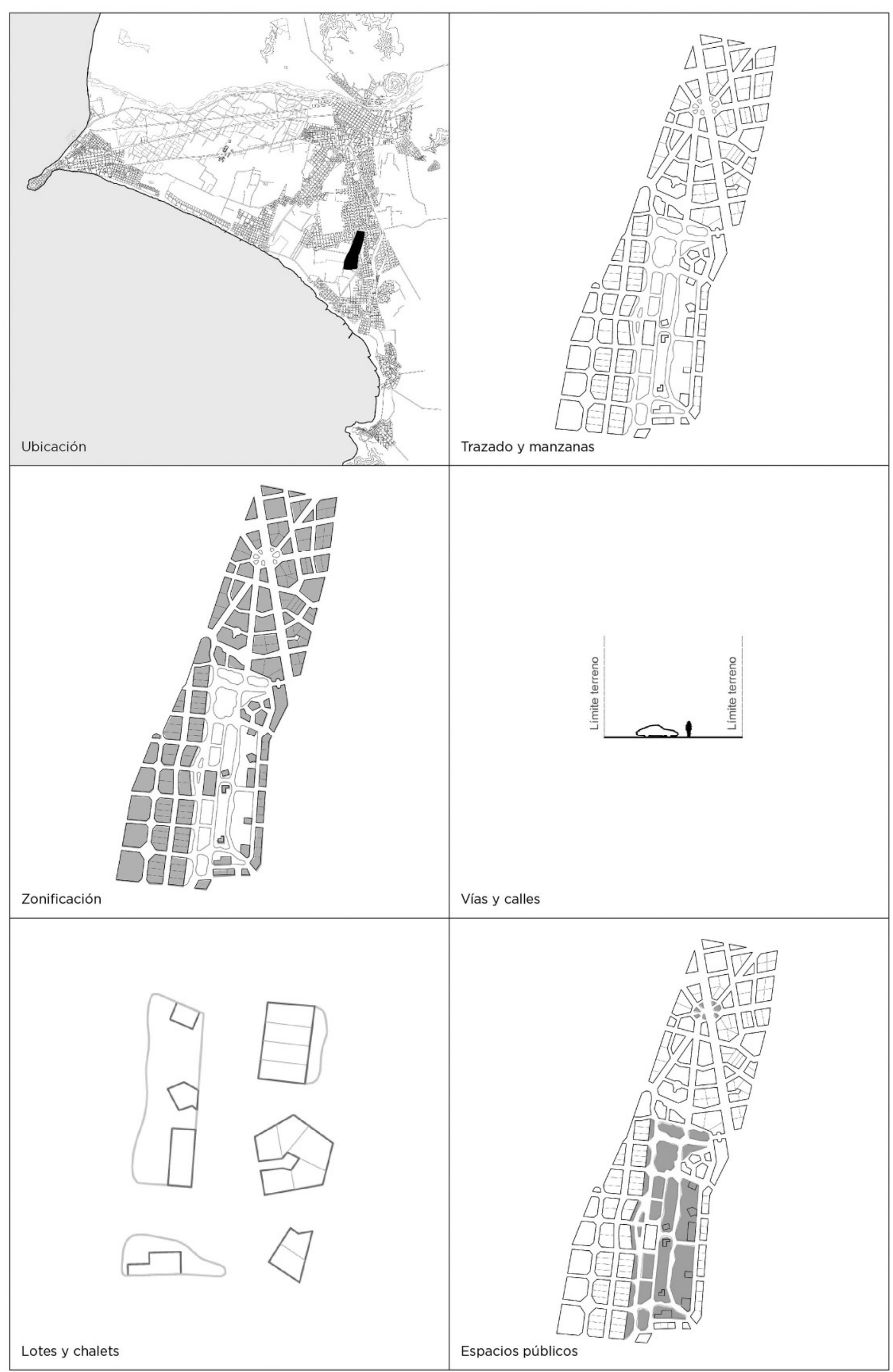

Figura 3. Urbanización San Isidro. Lima. 
La urbanización tuvo la privilegiada circunstancia de rodear al bosque del Olivar y estar atravesada por la avenida Leguía, actual avenida Arequipa. La avenida Leguía fue el principal eje articulador y de conexión de la urbanización con el centro de Lima y Miraflores. El bosque del Olivar pasó a ser el corazón de la urbanización y principal área verde, una de las más grandes que presentaba la ciudad, ya que la mayoría de las nuevas urbanizaciones buscaba maximizar la rentabilidad y el espacio destinado a la recreación en áreas verdes era casi inexistente. Inicialmente, el Olivar no fue utilizado como espacio público sino como espacio productivo. Hacia 1924, su principal función seguía siendo la productiva. Hoy es uno de los espacios verdes más importantes de la capital y cuenta con cerca de 1946 árboles en aproximadamente 10 hectáreas.

\subsection{Urbanización Chorrillos}

La urbanización Chorrillos cuenta con una historia que se remonta a la época prehispánica. Los pobladores de la cultura Ichma formaron el importante centro urbano Ichimatampi en las faldas del morro solar. Llegada la colonia española, el espacio se empezó a poblar inicialmente por pescadores y las construcciones fueron de carácter rústico. Durante la guerra del Pacífico, Chorrillos fue invadida y ocupada por las fuerzas chilenas durante dos años. En 1901 fue considerada como ciudad. En ella se proyectaron urbanizaciones como Chorrillos, Villa Olaya o La Chira, urbanizaciones que le pertenecieron a Juan Leguía Swayne, hijo del presidente Augusto B. Leguía, quien posteriormente fue juzgado junto a su padre.

La urbanización se ubicó frente a la antigua zona del distrito de Chorrillos y tiene como principales referentes al morro solar y la costa del Pacífico. Su principal vía de conexión con la ciudad fue la avenida Leguía, que a su vez la conectaba con la urbanización La Chira. Inicialmente, Chorrillos estaba conectada con el centro de Lima mediante las vías del ferrocarril y tenía como parada intermedia al distrito de Miraflores. (Figura 4)

\section{Trazado, manzanas y zonificación}

La forma de las manzanas trazadas en la urbanización Chorrillos son ortogonales (cuadradas o rectangulares). Las manzanas rectangulares se dispusieron al borde de la avenida Augusto B. Leguía y cuentan con medidas de 100 por 140 metros. Las manzanas cuadradas fueron rotadas $45^{\circ}$ respecto al eje de la organización y cuentan con una medida de 100 metros por lado. El trazado se organiza a partir de la avenida Leguía y siguió una grilla ortogonal, con la particularidad de manzanas rotadas que diferencian la urbanización. El trazo de Chorrillos también es intervenido por dos óvalos que deforman y recortan las manzanas que los circundan.

Al igual que la mayoría de urbanizaciones, Chorrillos fue pensada al inicio únicamente para uso de vivienda. Los habitantes del centro de Lima empezaron a migrar a los suburbios y la urbanización Chorrillos buscó satisfacer esa demanda. Actualmente, la zonificación ya no es exclusivamente residencial. Al igual que en todo Lima, la falta de espacios pensados para el comercio y el acelerado crecimiento demográfico, ha diversificado los usos y la mayoría de lotes han sido subdivididos horizontal y verticalmente para cumplir esta función.

\section{Vías, lotes y chalets}

La urbanización Chorrillos cuenta con dos secciones de vías: la de la avenida Leguía, con 38 metros de ancho; y la sección de las calles o vías secundarias, con aproximadamente 20 metros de ancho. A diferencia de la urbanización San Isidro, Chorrillos no llegó a realizar la pavimentación de sus calles. Sin embargo, se realizaron las obras de saneamiento e iluminación eléctrica exigidas por el Estado. Al haber manzanas rotadas $45^{\circ}$, se generaron calles que se bifurcan y esquinas que congregan la intersección de hasta cinco calles.

Los lotes proyectados en la urbanización Chorrillos presentaban diversidad de condiciones y tamaños. Hacia la avenida Leguía, se tenían lotes de un frente y en esquina. La urbanización no contempló lotes aislados ni con doble frente. Los tamaños fueron variados, entre 300 y $1000 \mathrm{~m} 2$ aproximadamente. Los más pequeños son los lotes alrededor de los óvalos, que están recortados hacia un lado de forma semicircular. En su mayoría, 


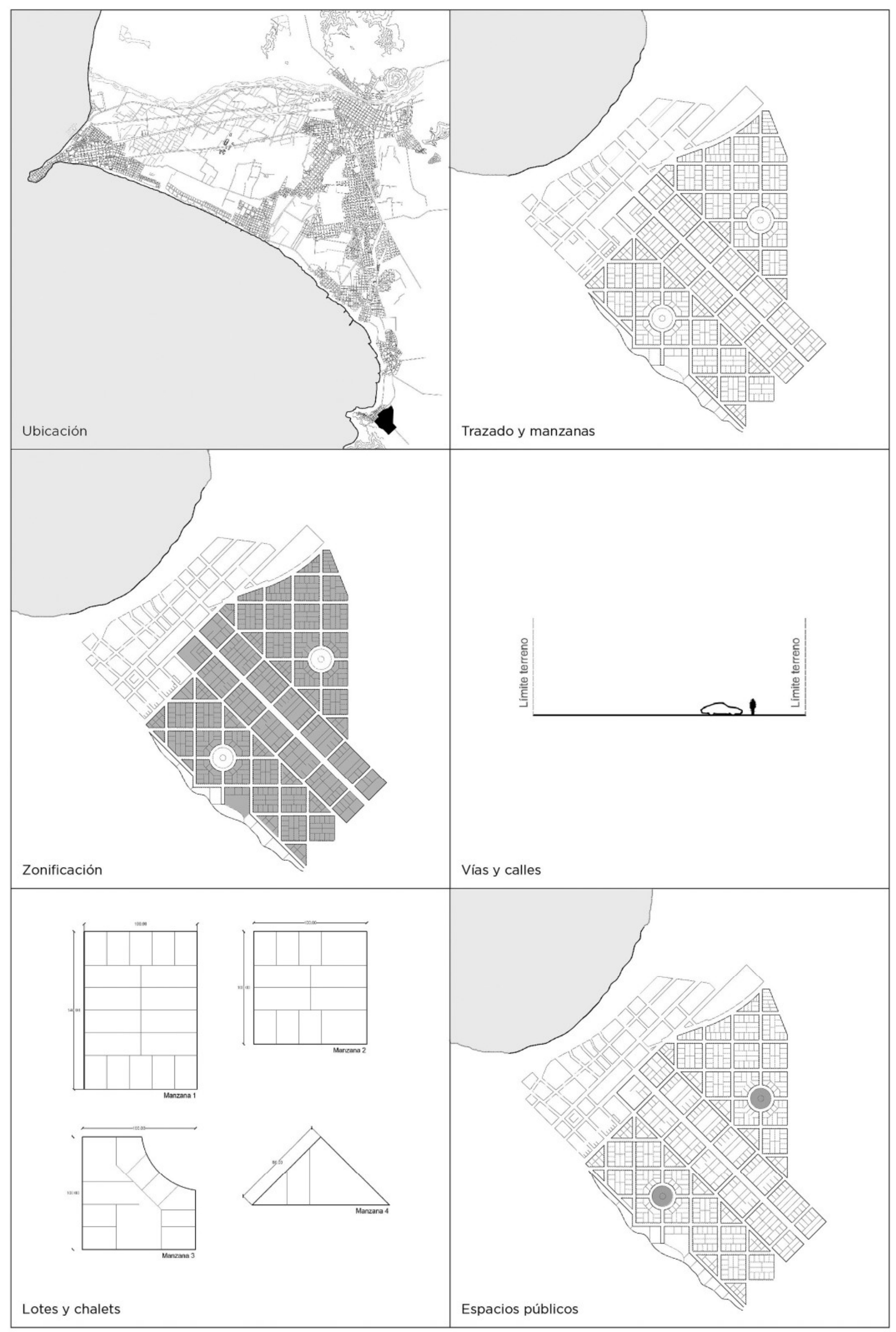

Figura 4. Urbanización Chorrillos. Lima. 
se ofrecieron únicamente terrenos, aunque también se muestran algunos anuncios de venta de chalets en la revista Ciudad y Campo. El costo de la venta de un terreno de 647 $\mathrm{m} 2$ fue de $2500 \mathrm{Lp}$ y los precios de 2 chalets, uno de $150 \mathrm{~m} 2$ con 7 habitaciones, baño, cocina, vestíbulo y jardín, y otro de $450 \mathrm{~m} 2$ con 5 dormitorios y baños, fue de $650 \mathrm{Lp}$ y $1400 \mathrm{Lp}$, respectivamente.

\section{Espacios públicos}

En la urbanización Chorrillos únicamente se proyectaron dos óvalos destinados a espacios públicos o áreas verdes, ambos están rodeados por vías y no se tiene acceso directo. El aspecto actual de los óvalos es parcial, ya que la urbanización tampoco se construyó en su totalidad. A diferencia de la urbanización Miramar, la urbanización Chorrillos sí proyectó espacios públicos, aunque estos espacios representan una pequeña fracción comparada al área total de la urbanización. Además, los óvalos no se pensaron como espacios de plaza o parque, simplemente fueron colocados en el plano como áreas verdes, sin ser concebidos para ser utilizadas como espacios públicos.

\subsection{Urbanización Miramar}

La urbanización Miramar se proyectó en el actual distrito de San Miguel, entre La Punta y Chorrillos. La urbanización fue concebida a partir de la consolidación de la entonces nueva avenida Costanera, inaugurada el 28 de octubre de 1928 y en conmemoración al Día del camino. El primer tramo de la avenida Costanera conectaba Bellavista con San Miguel y consolidó el desarrollo urbano al borde de las costas del océano Pacífico.

El trazo de la urbanización Miramar no llegó a realizarse en su totalidad. Se estima que, del trazo original, solo se consolidó la quinta parte. Actualmente, la urbanización Miramar es atravesada longitudinalmente por la avenida La Paz y la sección de esta vía se ha mantenido. La urbanización se caracteriza por ubicarse frente a la hoy Costa Verde y presentaba una forma alargada con tres filas paralelas de manzanas y lotes. (Figura 5)

\section{Trazado, manzanas y zonificación}

A diferencia de la urbanización San Isidro y Chorrillos, Miramar presentaba manzanas de mucha mayor área. Las manzanas ubicadas en la primera fila presentaban doble frente: uno de forma irregular y el otro hacia la hoy avenida La Paz. Las manzanas de la segunda fila tenían forma rectangular y según el trazo original no presentaban subdivisiones. Finalmente, la tercera fila presentaba manzanas de mucho menor tamaño y proporción. La organización de la urbanización parte de la avenida La Paz, de forma longitudinal y paralela a la costa.

Los pocos terrenos que llegaron a respetar el trazo original estaban destinados a vivienda. Los tamaño y proporciones fueron tan grandes que tuvieron que ser reducidos en años posteriores. Miramar es una de las pocas urbanizaciones que no llegó a consolidarse y su trazado original no se completó. A sus bordes se siguió usando el orden de grilla ortogonal y se respetó la avenida La Paz, aunque no el primer diseño.

\section{Vías, lotes y chalets}

Miramar presentaba dos tipos de secciones: la avenida La Paz, de aproximadamente 20 metros; y las avenidas o vías secundarias de aproximadamente 10 metros. La urbanización contó con obras de saneamiento y electricidad, mas no se llegó a pavimentar sus calles. El principal promotor y regulador de las nuevas urbanizaciones fue el Estado y se requería la instalación de servicios básicos de higiene en toda nueva urbanización. Actualmente, las secciones se han mantenido y la avenida La Paz se ha distribuido en cuatro carriles vehiculares y dos bermas laterales. Las vías perpendiculares no han sido interrumpidas y tienen salida al malecón y a la avenida Costanera.

En la urbanización Miramar no se proyectaron ni construyeron chalets para su venta. El área de los lotes variaba desde $600 \mathrm{~m} 2 \mathrm{a}$ más de 7000 m2. En la revista Ciudad y Campo se publicaron a la venta lotes de $600 \mathrm{m2}, 675$ $\mathrm{m} 2,1200 \mathrm{m2}, 1320 \mathrm{m2}, 3000 \mathrm{m2}, 4400 \mathrm{m2}$ y $7860 \mathrm{mz}$. Los terrenos estaban orientados a la clase media y los costos por metro cuadrado eran de entre 1.5 y 3 Lp. Los lotes de 


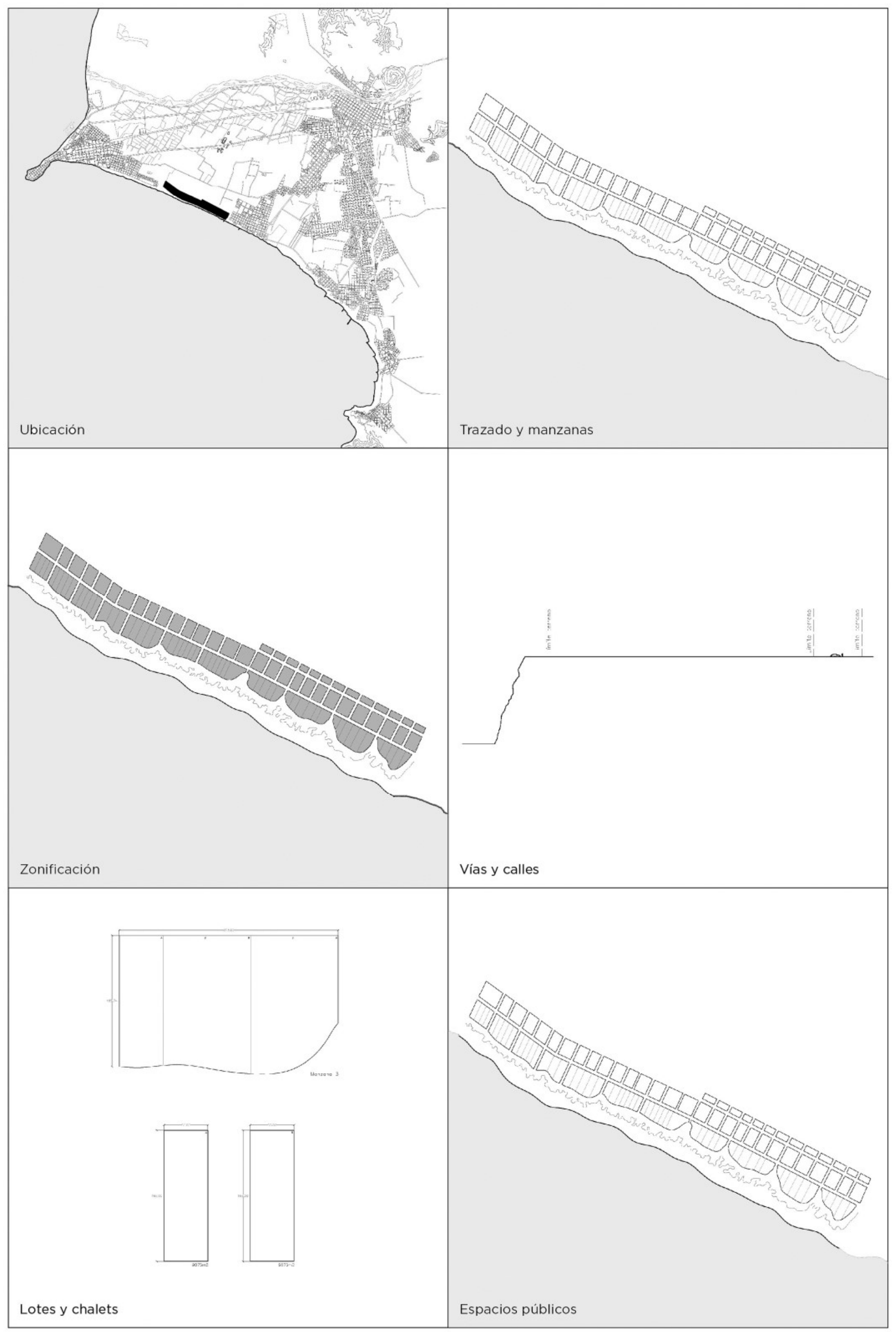

Figura 5. Urbanización Miramar. Lima. 
la urbanización Miramar tenían un costo muy bajo a diferencia de la urbanización Chorrillos y San Isidro. Chorrillos tenía un costo de 6.5 Lp por $\mathrm{m} 2$ aproximadamente, mientras que en San Isidro el costo podía elevarse a más de 10 Lp por m2. La ubicación, el entorno, la falta de pavimentación y el poco desarrollo urbano colindante, generaron que los costos de Miramar se encuentren por debajo de las mencionadas urbanizaciones.

\section{Espacios públicos}

De las tres urbanizaciones seleccionadas, Miramar no contempló áreas destinada a espacio público o zonas verdes. El espacio en su totalidad estaba lotizado y subdivido para que aumenten las ganancias y se aproveche al máximo los metros cuadrados existentes. A diferencia de la urbanización San Isidro, Miramar no contaba con algún parque o bosque previo como El Olivar. Tampoco se proyectó ni un ovalo como en el caso de la urbanización Chorrillos. Miramar tenía la ventaja y privilegio, a diferencia de San Isidro y Chorrillos, de estar al borde de la costa del Pacífico, por lo que pudo haber proyectado algún importante malecón que genere un espacio público y de encuentro para los ciudadanos.

\section{Conclusiones}

Lima fue el principal espacio de intervención constructiva y desarrollo urbano durante el segundo gobierno de Leguía. Las más importantes obras públicas, saneamiento, infraestructura y urbanización, se desarrollaron con la intención de convertir a la capital peruana en la ciudad más importante de la costa del Pacífico sur. Durante este periodo, a partir de la construcción de las avenidas El Progreso, Leguía y la Costanera, se desarrollaron más de treinta urbanizaciones en el triángulo conformado entre Lima, Callao y Chorrillos.

Las nuevas urbanizaciones, en su mayoría, fueron previamente haciendas pertenecientes a miembros de la oligarquía limeña. Se estableció una nueva forma de generar negocios a partir de la venta de terrenos y chalets, buscando optimizar y hacer más rentable cada urbanización. Las urbanizaciones fueron pensadas en su totalidad para el uso de vivienda, ya que el centro de poder comercial y financiero se seguía manteniendo en el centro de Lima. El nuevo "estilo de vida" estuvo definido a partir del automóvil, que conectaba la zona de vivienda con el centro. Las nuevas urbanizaciones buscaron satisfacer las necesidades de habitación surgidas a partir del incremento de la población en la capital.

La urbanización San Isidro fue de las más aristocráticas y destinada a clases altas. El trazado no sigue la grilla cuadriculada y está configurada, más bien, por calles y vías curvas, diagonales, largas y cortas. La urbanización San Isidro representa uno de los capítulos más significativos de la historia urbanística de Lima y es considerada un hito de ruptura (Ludeña, 2009). El bosque del Olivar fue el principal atractivo y su presencia como espacio público y área verde para la ciudad aún se mantiene hasta la actualidad.

La urbanización Chorrillos fue proyectada por encargo del hijo del presidente Leguía y tiene como principales referencias al morro solar y el malecón de Chorrillos. Las manzanas hacia la avenida Augusto B. Leguía, hoy avenida Huaylas, tenían forma rectangular, mientras que las posteriores presentaban un giro de 45 grados y forma cuadrada. La avenida A. B. Leguía fue la conexión con la urbanización la Chira y las secciones variaban entre veinte y cuarenta metros. Esta urbanización no se llegó a construir en su totalidad y las secciones de las vías se mantienen hasta el momento. Hoy los grandes lotes han sido subdivididos, el distrito ha aumentado su densidad y se ha modificado los usos. La urbanización no dejó espacio público y las pocas áreas verdes de los óvalos no fueron respetadas.

La urbanización Miramar se encuentra en el hoy distrito de San Miguel, frente al océano Pacífico. A diferencia de la urbanización San Isidro y Chorrillos, Miramar presenta un trazado alargado con doble y triple fila de terrenos. La urbanización está atravesada por la avenida La Paz y los lotes cuentan con tamaños de hasta $8000 \mathrm{m2}$. Miramar fue una urbanización que no contempló área libre destinada a uso público o áreas verdes. Se buscó la máxima rentabilidad con la venta de grandes lotes y manzanas. Actualmente se mantiene las secciones de las vías y los lotes han sido ocupados en su totalidad. 
El trazado de las urbanizaciones desarrolladas durante el segundo gobierno de Leguía constituye la base de nuestra capital. La especulación y la búsqueda del máximo de ganancias no permitieron que las urbanizaciones proporcionen grandes espacios públicos ni importantes áreas verdes. La actual ausencia de espacios públicos para el ciuda- dano, plazas, parques, bosques y espacios de recreación en la capital, puede explicarse, en parte, en el tipo de urbanismo y forma de vida que se fomentó en esta determinante etapa del desarrollo de nuestra ciudad. La ausencia no pudo ser remediada en años posteriores y actualmente Lima adolece de espacios de afirmación e identificación ciudadana.

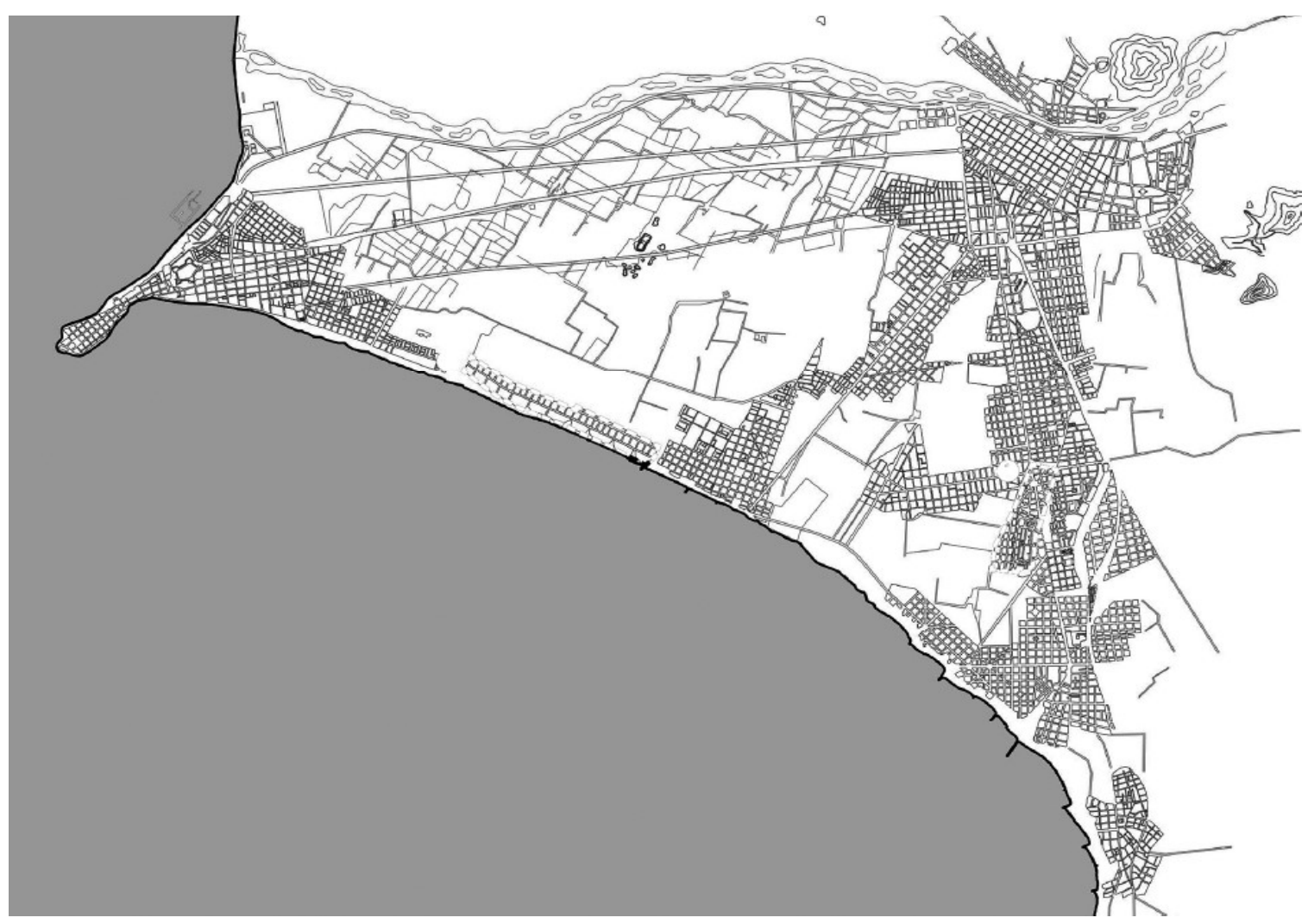

\section{Referencias bibliográficas}

Basadre, J. (2005). Séptimo periodo. El Oncenio (19191930). En: Historia de la República (1822-1933). Tomo 14. Lima: El Comercio.

Bromley, J. \& Barbagelata, J. (1945). Evolución urbana de la ciudad de Lima. Lima: Lumen.

Clayton, L. (2002). Las relaciones peruano-estadounidenses desde la independencia hasta el siglo XX. Lima: CEPEI.

Ciudad y Campo (1925) La urbanización de San Isidro (octubre). Ciudad y Campo, 2(11), 21-23.

-(1926) Estudios del Ensanchamiento de Lima (junio). Ciudad y Campo, 3(19), 37-39.

Gunther, J. (1983). Planos de Lima 1613-1983. Lima: Municipalidad de Lima.

Hamann, J. (2015). Leguía, el Centenario y sus monumentos. Lima: 1919-1930. Lima: Fondo editorial Pontificia Universidad Católica del Perú.
Harth-terré, E. (1935). Lima contemporánea. Lima en el IV centenario de su fundación: Monografía del departamento de Lima. Lima: Minerva.

Ludeña, W. (2002). Lima: poder, centro y centralidad: Del centro nativo al centro neoliberal. EURE (Santiago), 28(83), 45-65. Recuperado de https://dx.doi. org/10.4067/S0250-71612002008300004

Ludeña, W. (2009). Urbanismo Dixit: Inquisiciones. Quito: Organización Latinoamericana y del Caribe de Centros Históricos.

Mattos Cárdenas, L. (2004). Urbanismo andino e hispanoamericano. Ideas y realizaciones (1530-1830). Lima: Fondo editorial Universidad Nacional de Ingeniería.

Ortiz de Zevallos, A. (1992). Urbanismo para sobrevivir en Lima. Lima: APOYO. 